\title{
Francis Carter, Peter Jordan et Violette Rey (dir.), 1998, Central Europe after the fall of the Iron Curtain - Geopolitical perspectives, spatial patterns and trends
} Coll. «Wiener Osteuropa-Studien », Éd. Peter Lang, Europäischer Verlag der Wissenschaften, Francfort / Berlin / Berne / New York / Paris / Vienne, $341 \mathrm{p}$.

\section{Gabriel Wackermann}

\section{(2) OpenEdition}

\section{Journals}

Édition électronique

URL : http://journals.openedition.org/rge/4373

DOI : $10.4000 /$ rge. 4373

ISSN : 2108-6478

Éditeur

Association des géographes de l'Est

Édition imprimée

Date de publication : 1 septembre 1999

ISSN : 0035-3213

Référence électronique

Gabriel Wackermann, « Francis Carter, Peter Jordan et Violette Rey (dir.), 1998, Central Europe after the fall of the Iron Curtain - Geopolitical perspectives, spatial patterns and trends », Revue Géographique de l'Est [En ligne], vol. 39 / 4 | 1999, mis en ligne le 03 septembre 2013, consulté le 25 septembre 2020. URL http://journals.openedition.org/rge/4373; DOI : https://doi.org/10.4000/rge.4373

Ce document a été généré automatiquement le 25 septembre 2020

Tous droits réservés 


\section{Francis Carter, Peter Jordan et Violette Rey (dir.), 1998, Central Europe after the fall of the Iron Curtain-Geopolitical perspectives, spatial patterns and trends}

Coll. « Wiener Osteuropa-Studien », Éd. Peter Lang, Europäischer Verlag der Wissenschaften, Francfort / Berlin / Berne / New York / Paris / Vienne, $341 \mathrm{p}$.

Gabriel Wackermann

\section{RÉFÉRENCE}

Francis Carter, Peter Jordan et Violette Rey (dir.), 1998, Central Europe after the fall of the Iron Curtain - Geopolitical perspectives, spatial patterns and trends, Coll. « Wiener OsteuropaStudien », Éd. Peter Lang, Europäischer Verlag der Wissenschaften, Francfort / Berlin / Berne / New York / Paris / Vienne, 341 p.

1 L'ouvrage s'appuie sur des textes présentés en 1994 lors du congrès de l'Union géographique internationale de Prague. Composée entièrement en langue anglaise par 23 auteurs, dont quatre géographes français, parmi lesquels les deux spécialistes chevronnées Marie-Claude Maurel et Violette Rey, cette publication est structurée autour d'une présentation globale des perspectives géopolitiques, puis d'une analyse des principaux processus de transformation, avant de livrer trois exemples « régionaux » (Frioul-Vénétie Julienne, Pologne et Autriche), ainsi que des modèles de migrations internationales internes à cet espace. Il s'agit déjà d'une seconde édition justifiée par l'important succès de la précédente en un si court laps de temps. 
2 La profusion d'ouvrages édités depuis le début de la décennie actuelle sur l'Europe centrale ou médiane, notamment sur l'Europe-ex-collectiviste, devrait a priori commencer à tarir la source d'inspiration. Il n'en est rien, car cet espace complexe constitue à la fois un terrain d'observation remarquable des transformations inhérentes à la chute du collectivisme et à la mise en place consécutive, cahin-caha, d'une économie de marché, ainsi qu'une excellente occasion de renouvellement ou d'affinement des méthodes d'approche méthodologique compte tenu des multiples problèmes inédits soulevés par la grande mutation intervenue. L'ouvrage présenté par ses trois co-directeurs a le mérite de constituer même en 1998 un apport original à la connaissance des milieux socio-spatiaux concernés, notamment, comme l'indique son sous-titre, par son approche géopolitique des perspectives d'évolution. F. Carter, P. Jordan et V. Rey s'interrogent une fois de plus, et de manière très judicieuse, sur la pertinence de la notion d'Europe centrale et plus encore de celle relative à la «nouvelle Europe centrale ", évoquant à ce sujet la mémoire du très lucide et pragmatique géographe autrichien, mon ami Karl Sinnhuber, dont j'ai pu apprécier à mainte reprises la finesse d'analyse d'un espace qu'il connaissait et pratiquait constamment, depuis son adolescence assombrie par l'annexion nazie des territoires étudiés, en passant par la période soviétique, jusqu'à l'actuelle période dite de transition. Tandis que des États nouveaux tels que la Slovénie, la Croatie ou la Slovaquie sont susceptibles d'alimenter la réflexion sur la nouvelle Europe centrale, la Hongrie et la Pologne, candidates à l'entrée dans la très occidentale Union européenne, constituent des espaces intermédiaires, des ponts entre l'Ouest et l'Est. Les actuels mouvements migratoires en provenance de l'ancienne Union soviétique rappellent toutefois constamment la position géopolitique de l'Europe centrale en tant que centre et interface. La haute densité en lignes de rupture et discontinuités spatiales héritée de l'Histoire et confirmée par les tracés politiques récents tempère en outre le passage d'ouest en est.

Quoique les contributions soient de valeur inégale, leur variété d'origine et leur nombre illustrent la diversité des aspects de portée générale et des facettes régionales ou intrarégionales inhérentes à l'espace étudié. L'un des intérêts de l'ouvrage réside dans le fait que les réflexions géopolitiques ont été étroitement associées à l'examen des flux supranationaux générés par la période de transition. Le livre montre également combien les traditionnelles limites de l'Europe centrale sont dépassées dans les faits par des débordements en direction de l'Est, du Sud-est et du Sud. Le terme d'Europe centrale est au fond devenu un usage commode à des fins politiques, incluant autant les demandes d'aides financières que des appels à l'investissement. La question demeure posée de savoir si l'Europe centrale a des caractéristiques spécifiques et, dans l'affirmative, en quoi celles-ci consistent. Il faudrait alors aussi circonscrire le centre, le « cœur» de cet ensemble spatial.

4 La plupart des auteurs militent davantage en faveur de la non-existence de l'Europe centrale que de son existence, d'autant plus que cette aire n'a ni unité politique ni unité économique. Elle est au contraire divisée en États centripètes dont chacun cherche individuellement sa voie du côté de l'Union européenne. Aucun des auteurs ne reconnaît ainsi une Europe centrale au sens politique ou économique du terme. Si l'Histoire a marqué cet espace par des empreintes culturelles spécifiques et complexes, il n'est pas évident qu'il en soit ainsi dans le temps présent.

5 Les positions critiques prises par d'authentiques compétences endo- et exogènes confèrent à cette publication une valeur primordiale. Elles rejoignent celles de 
plusieurs de nos collègues allemands de l'Ouest qui, dès 1989, ont refusé, dans le cadre de la Conférence de géographes franco- et germanophones que j'ai eu l'occasion de mettre sur pied durant la fin de la guerre froide avec mon collègue allemand Wolfgang Brücher, en accord avec les Comités nationaux de géographie des deux pays, à la fois la notion de «Mitteleuropa» et d'«Europe centrale». Nous voici invités depuis longtemps à une prudence constante qui tranche une fois de plus avec les affirmations idéologiques ou médiatiques, de quelque nature que ce soit. 